\title{
Lower Computation and Storage Complexity of QC-LDPC Codes in Rayleigh Fading Channel
}

\author{
Abid Yahya, Othman Sidek, M. F. M. Salleh and Farid Ghani
}

\begin{abstract}
This paper presents the construction of large girth Quasi-Cyclic low density parity check (QC-LDPC) codes and a new multicarrier frequency hopping spread spectrum (MCFH-SS) system. The performance of newly obtained codes is evaluated by comparing with Reed Solomon (RS) codes. The computer simulation results show that the two girth-twelve QC-LDPC codes significantly outperform conventional RS codes with a gain of $2.1 \mathrm{~dB}$ at
\end{abstract}

Index Terms-Bit error rate, DPSK, Girth, QC-LDPC, Rayleigh fading

\section{INTRODUCTION}

A thought-provoking mission for wireless channels to communicate authentic data postulates many unusual problems. Low-density parity-check codes [1] have acquired considerable attention due to its near-capacity error execution and powerful channel coding technique with an adequately long codeword length. The performance of LDPC codes has been investigated in [2, 3], and are encountered to outperform turbo codes.

The potency of LDPC codes is outclass over the additive White Gaussian noise (AWGN) channel [4], where coherent detection employing phase shift keying (PSK)[5] can be carried out with carrier phase estimation. Precise phase tracking and high-quality estimation of channel state information (CSI) is required for the appropriate execution of coherent detection.

The coherent detection of PSK often experiences problems while performing on Rayleigh fading channels. The reason is inaccurate CSI, due to complex channel gains variation with a passage of time, specified that LDPC codes characteristically function at a very low signal-to-noise ratio (SNR).

In order to acquire accurate CSI, the authors in [6], have derived the estimates of the complex channel gain and variance of the additive noise from known pilot symbols and an estimation filter. In their work the authors have employed Turbo codes which have been detected coherently over for

Manuscript received October 9, 2008

Abid Yahya Abid Yahya is with the Collaborative Microelectronic Design Excellence Centre Universiti Sains Malaysia (phone: +6045995805; fax: +6045930920 )

Othman Sidek is the director of Collaborative Microelectronic Design Excellence Centre Universiti Sains Malaysia.

M. F. M. Salleh is with the School of Electrical and Electronic Engineering, Universiti Sains Malaysia.

Farid Ghani is currently working as Professor in the School of Computer and Communication Engineering, Universiti Malaysia Perlis, Malaysia. transmission.

Other authors have designed three capacity approaching LDPC codes having CSI at transmitter and receiver. In [7], authors have used extrinsic information transfer (EXIT) function method to design the LDPC codes in the SNR regime for flat Rayleigh fading channels by employing quadrature phase-shift keying (QPSK) modulation. The power consumption problem arises again, because the QPSK modulation uses twice the power of PSK, since two bits are transmitted simultaneously.

Wireless communications are impacted by respective channel imperfectness that considerably degrades system execution. One of the aftermaths is the loss of carrier phase synchronization. Differential and noncoherent techniques do not entail a phase reference and, for this reason, have acquired esteem recognition in wireless communications

To avoid CSI, some authors [8] have employed frequency shift keying (FSK) modulation scheme for noncoherent detection with LDPC codes. Nevertheless, the power efficiency of FSK is less than PSK.

No channel estimation or equalization is expected if DPSK modulation is employed. Accordingly, the receiver can be more elementary and pilot symbols can be neglected at the cost of higher SNR.

In $[9,10]$, the authors have prevailed over the CSI by employing differential PSK modulation over Rayleigh fading channels with regular LDPC codes. However, the metric, which has been derived in [9], requires accurate estimates of the amplitudes of the complex channel gains, which can degrade the performance of the system if estimation falls erroneous.

In [10], authors have emphasized the operation of differential detection relatively to codes themselves. They have employed LDPC codes with column weights 3 and 4, which have high computation and storage complexity. While LDPC codes with column weight ${ }^{j}=2$, have respective advantage, since they have lower computation and storage complexity and their encoders and decoders are merer to employ. In work [9, 10], authors have used differential PSK modulation but with regular LDPC codes.

In this work, large girth QC-LDPC codes have been developed by deriving the basic idea from Bit-Filling (BF) and Progressive Edge-Growth algorithms proposed by Campello et al. (2001) [11] and $\mathrm{Hu}$ et al. (2005)[12], respectively. Some more constraints have been employed in this work by keeping the row weight dependable on group size. A degree of distribution is designed carefully, since a degree of distribution particularly variable-node degree distribution, deeply affects the error correcting performance. 
A degree of distribution is said to be concentrated if every node has the same degree.

\section{PROPOSED FOR PAPER SUBMISSION}

The bit-filling (BF) algorithm designs a LDPC code by connecting rows and columns of a code one at a time without violating the girth condition. In the $\mathrm{BF}$ algorithm, the number of row connections is almost uniformly distributed by first selecting randomly the rows with the least number of connections. The codes so obtained are either with a fixed row or fixed column weight. The structure of row-column connections in the BF algorithm is, therefore, almost random and hence increases the complexity of the decoder. In order to simplify the hardware implementation, the BF algorithm must be modified to incorporate some form of structured decoder interconnections. In the proposed algorithm this structuring in interconnections is brought about by dividing the rows with respect to the group size. Such a division guarantees a concentrated node degree distribution and reduces the hardware complexity.

The proposed algorithm for the construction of a $(\mathrm{N}, \mathrm{j}, \mathrm{k})$ code is best described in the form of following steps.

1. Rows in the set could be chosen randomly or sequentially, preferable choice is random since random searches will result in a variety of codes.

2. The rows are evenly divided with respect to the group size. Each group must contain k rows. The row groups are paired in such a way that each group appears k times so that there are $2 \mathrm{k}$ row group pairs.

3. Note: The dependence of row weight on group size keeps the constructed codes regular. Otherwise the groups with different number of appearances will generate irregular codes.

4. Rows in the second and following groups are placed in descending order; this satisfies the least desired distance to search, for each group.

As an example Figure 1 shows row connections for a $(16,2$, 4) LDPC code construction using steps 1 to 3 above. There are two groups of size 4 . The first group and row 1 are always chosen as the reference group and row, respectively. The Girth of this code is found to be eight.
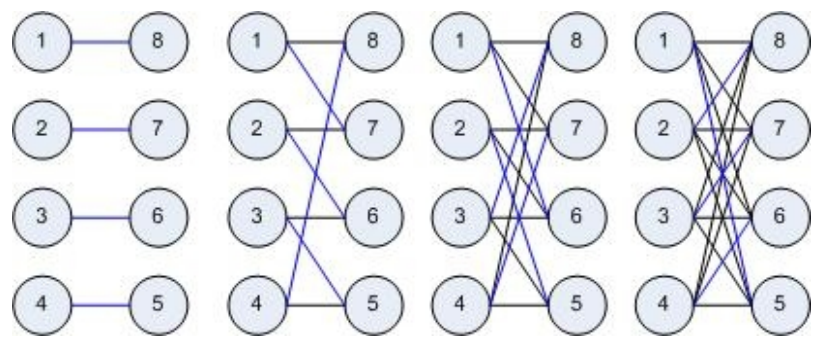

Fig.1 Graphical representation of a $(16,2,4)$ code

With reference to Figure 1 the following may be noted:

5. The regular codes make it easy to construct the parity check matrix due to uniform distribution of 1's and 0's from the column formation.

6. The row groups are paired two times the row weight, which has cut down hardware implementation cost and complexity as compared to the connection of individual columns and rows. The complexity of directing within groups computes on the transposition employed to connect rows and columns between groups. This modifies handling, when messages are communicated between functioning nodes.

It may also be pointed out that:

7. Adopting Algebraic methods [13], are used to determine bounds on girth, rate or code dimensions for QC-LDPC codes.

8. Rows are used to form a distance graph, which is then transformed to a parity-check matrix. To acquire a given girth, rows that are at desired distance from each other are searched sequentially or randomly in each group and connected.

9. QC-LDPC codes have encoding advantage over conventional LDPC codes and their encoding can be carried out by shift register with complexity linearly proportional to the number of parity bits of the code. Additionally, QC-LDPC codes require less amount of memory as compared to general LDPC codes, since their parity check matrices consist of circulant permutation matrices or the zero matrix. Actually, their expected memory for storing them can be cut down by a factor $\frac{1}{p}$, when $p \times p$ circulant permutation matrices are employed. The entire parity check matrix can be partitioned into an array $p \times p$ block matrices, each one denoted as $H_{i, j}$, each block matrix $H_{i, j}$ is either a zero matrix or right cyclic shift of an identify matrix.

10. For each, decoding iteration, to be accomplished, $2 p$ clock cycles are the requisite. The decoder works in check node and variable node processing mode during the first and second $p$ clock cycles respectively. The decoder performs the computations of all the check node and variable nodes and brings in the message passing between neighboring nodes.

\section{RESUlTS AND DISCUSSION}

The focal intention of the algorithm is to generate high-rate LDPC codes specified a particular code length

The allowable maximum number of iteration for decoder is set to 60 with 5 microseconds symbol period and 1.1 milliseconds coherence time in a flat Rayleigh fading environment by employing DPSK modulation scheme.

The curves in Figure 2 show slight performance enhancement at diversity levels $\mathrm{L}=2$ and $\mathrm{L}=3$. However, by increasing the diversity level to $\mathrm{L}=4$ the newly obtained codes outperform the results obtained in Figure 6 of [9], by about $1.5 \mathrm{~dB}$ gain at $10^{-5}$ BER.

LDPC codes with column weight $j=2$, have respective advantage, since they have lower computation complexity and storage complexity, their encoders and decoders are merer to employ. They have ameliorated block error statistics properties which have been mentioned by Song et al. in [14].

The performance of two twelve-girth QC-LDPC against 
RS codes in PBNJ environment over AWGN channel is revealed in Fig.3. The signal noise ratio is set to be $20 \mathrm{~dB}$ to attain a packet error ratio of $10^{-3}$ against the value fractional bandwidth. The curves in Fig.3 show that newly obtained codes cater a gain of $2.1 \mathrm{~dB}$ compared with RS codes at the worst condition of partial band $\rho=1$.

This shows the robustness of the system by employing newly obtained codes as FEC code.

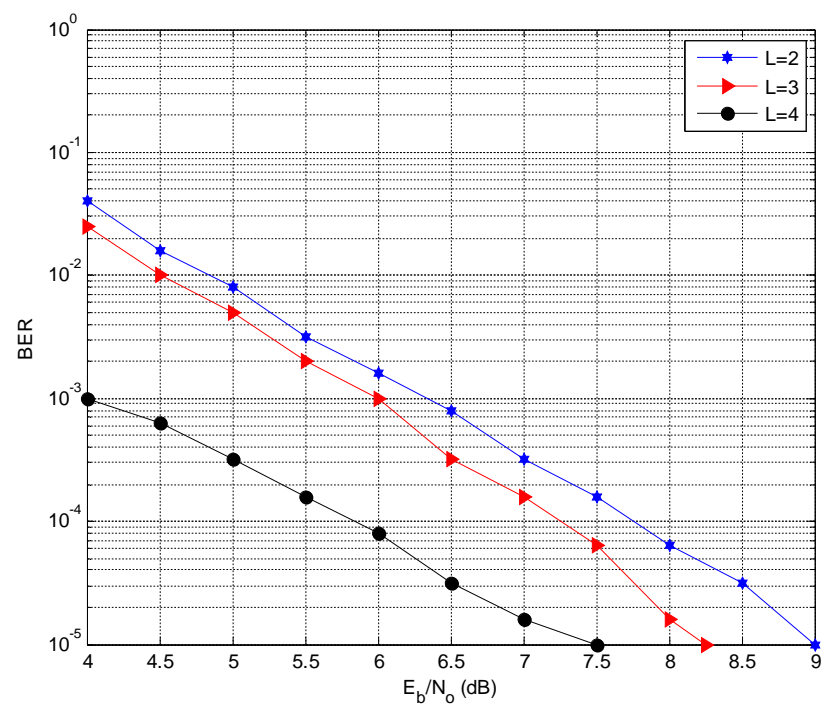

Fig. 2 BER of two girth-twelve QC-LDPC codes for given value of $E_{b} / N_{o}$ in Rayleigh fading channels at diversity levels 2, 3 and 4

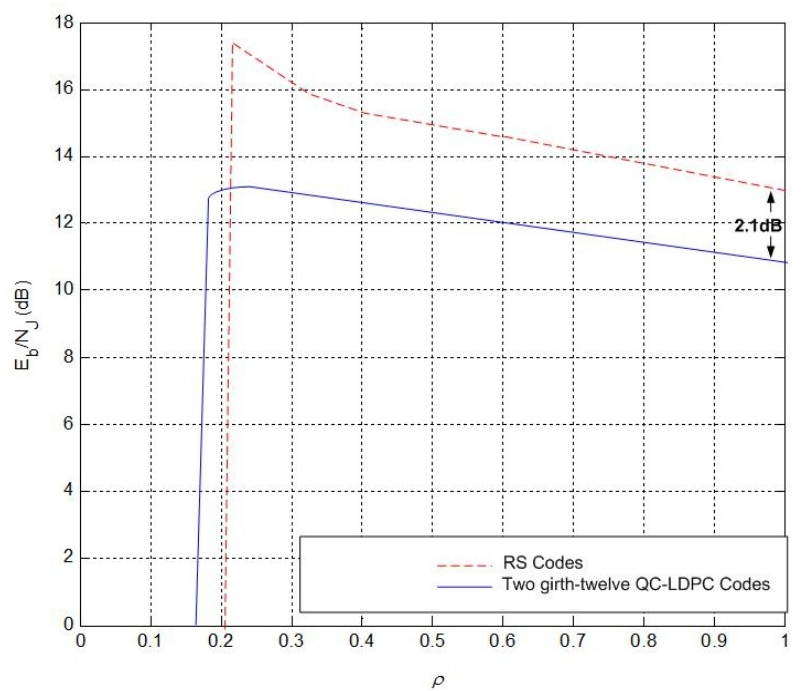

Fig. 3 Performance evaluation of two girth-twelve QC-LDPC codes and conventional RS codes over PBNJ environment

\section{REFERENCES}

[1] C. Berrou, A. Glavieux, and P. Thitimajshima, "Near Shannon limit error correcting coding and decoding: turbo-codes," in Proc. of IEEE ICC'93, (Geneva, Switzerland), May 1993, pp. 1064-1070.

[2] R. G. Gallager, Low Density Parity Check Codes. Research Monograph series, Cambridge: MIT Press, 1963.

[3] D. J. Mackay, "Good error-correcting codes based on very sparse matrices," IEEE Trans. Inform. Theory, Vol. 45, Mar. 1999, pp. 399-431.

[4] G. Caire and S. Shamai, "On the capacity of some channels with channel state information," IEEE Transactions on Information Theory, Vol. 45, no. 6,1999, pp. 2007-2019.
[5] M. C. Valenti, and Woerner, "Iterative Channel Estimation and Decoding of Pilot Symbol Assisted Turbo Codes over Flat-Fading Channels," IEEE Journal on Selected Area in Communications, Vol.19, no.9, 2001, pp.1697-1705.

[6] M. C. Valenti and B. D. Woerner, "Iterative Channel Estimation and Decoding of Pilot Symbol Assisted Turbo Codes Over Flat-Fading Channels," IEEE Journal on Selected Area in Communications, Vol. 19, No. 9, Sep 2001.

[7] Y. Jiang, A. Ashikhmin, and N. Sharma, "LDPC Codes for Flat Rayleigh Fading Channels with Channel Side Information," IEEE Transactions On Communications, Vol. 56, no. 8, August 2008.

[8] S. Supakwong and S. G. Wilson, "LDPC Coded M-ary Orthogonal Signaling with Noncoherent Detection," 2005 Conference on Information Sciences and Systems, The Johns Hopkins University, March 2005

[9] H. Tatsunami, K. Ishibashi, and H. Ochiai, "On the Performance of LDPC Codes with Differential Detection over Rayleigh Fading Channels," Proceedings IEEE, Vehicular Technology Conference, VTC 2006-Spring. Vol. 5, 2006 pp. 2388 - 2392.

[10] V. T. Nam, P. Y. Kam, and Y. Xin, "LDPC Codes with BDPSK and Differential Detection over Flat Rayleigh Fading Channels," Proceedings IEEE, Global Telecommunications Conference, 2007. GLOBECOM '07. Nov. 2007 pp. 3245- 3249.

[11] J.Campello, D.S. Modha, and S.Rajagopalan, " Designing LDPC codes using bit-filling," Proceedings of IEEE International Conference on Communications (ICC '01),' Helsinki, Finland, p.55-59, 2001.

[12] X.Y. Hu, E. Eleftheriouand and D.M. Arnold, " Regular and irregular progressive edge-growth tanner graphs," IEEE Transactions on Information Theory, vol. 51(1), p. 386-398, 2005.

[13] Y.Wang, J. S. Yedidia, and S. C. Draper, "Construction of high-girth QC-LDPC codes," 5th International Symposium on Turbo Codes and Related Topics, p.180-185, 2008.

[14] H. Song, J. Liu, and B. V. Kumar, "Low complexity ldpc codes for magnetic recording," IEEE Globecom 2002, Taipei, Taiwan, R.O.C., Nov 2002.

Abid Yahya received his B.Sc. degree from University of Engineering and Technology, Peshawar, Pakistan in Electrical and Electronic Engineering majoring in telecommunication. He completed his M.Sc. from school of Electrical and Electronic Engineering Universiti Sains Malaysia, and awarded graduate fellowship the M.Sc. research. He served as a lecturer at Multimedia University Malaysia. Currently he is working as a technical editor and Research Officer while pursing his Ph.D. at Universiti Sains Malaysia. His research areas include Wireless \& Mobile communication, interference and jamming rejection.

Othman Sidek obtained B.Sc. in electronics in 1982 at University Sains Malaysia (USM), M.Sc. in communication engineering from University Manchester Institute of Science and Technology, Manchester, United Kingdom in 1984. His topic for PhD was using AI in CAD for VLSI. His research interest includes advanced FPGA design, IC design and MEMS. Currently he is the director of Collaborative Microelectronic Design Excellence Centre, an initiative he started with the support of the Malaysian government in order to create more IC designers among Malaysian universities. Also actively involves with research supervision and publications.

Mohd Fadzli, Mohd Salleh received his B.Sc. degree in Electrical Engineering from Polytechnic University, Brooklyn, New York, U.S., in 1995. He was then a Software Engineer at MOTOROLA Penang, Malaysia, in R\&D Department until July 2001. He obtained his M.Sc. degree in Communication Engineering from UMIST, Manchester, U.K., in 2002. He completed his Ph.D. degree in image and video coding for mobile applications, in June 2006 from the Institute for Communications and Signal Processing (ICSP), University of Strathclyde, Glasgow, U.K. He is a member of IEEE.

Farid Ghani received B.Sc. ( Engg.) in Electrical Engineering, M.Sc. (Engg.) in Measurement and Control from Aligarh Muslim University, India and M.Sc. in Digital Signal Processing and Ph.D. in Digital Communication Systems from Loughborough University Of Technology (U.K). Dr. Ghani started his carrier as lecturer in the Department of Electronics Engineering, Aligarh Muslim University. In 1982 he was appointed as Professor in the same Department where he worked till 2004. From 1986 to 1991, he worked as Professor and Head of the Department of Electronics Engineering, Engineering Academy (Al-Fateh University), Libya, and again from 2004 to 2007 as Professor at Universiti Sains Malaysia. Professor Ghani is currently working as Professor in the School of Computer and Communication Engineering, Universiti Malaysia Perlis, Malaysia. His professional experience and interest are in the general areas of digital communication and digital signal processing, and more specifically in high 
speed data communication systems, mobile and wireless systems, radar detection, image and speech coding, and system identification techniques. He has a large number of publications to his credit and is he reviewer for several international research journals. He has extensively traveled abroad in connection with visiting assignments, invited lectures and conferences. Professor Ghani is Fellow of IET (UK), Fellow of Institution of Electronics and Telecommunication Engineers (India) and Fellow of National Telematic Forum (India). He is also registered with the Council of Engineers (UK) as Chartered Engineer 